\title{
Through the eyes of the Informationist: Identifying information needs of the Breast Imaging Service at a tertiary medical center specializing in cancer
}

\author{
Antonio P DeRosa, Donna S Gibson \\ and Elizabeth A Morris \\ Memorial Sloan Kettering Cancer Center Library, USA
}

\begin{abstract}
The information services offered by Embedded Librarians over the years have led to the more modern-and domain knowledge-specific - role of the Informationist. A 10-point questionnaire was developed and used to interview 12 attending physicians and three fellows chosen at random. The participants are either on the research track $(n=3)$ or the clinical track $(n=9)$. A two-part schematic was also created to capture more detailed feedback about the information needs and information-seeking behavior of clinicians regarding patient care (clinical) and research activities. Bibliographic management tool use and time-related factors were also captured in the interviews and written schematics. The role of the Informationist is an emerging, yet valuable one to assigned clinical groups. Clinician's knowledge-base, current awareness, productivity, and evidence-based care can be improved by use of Informationist services.
\end{abstract}

\section{Keywords}

bibliographic management, clinical medical librarian, embedded librarian, health information databases, Informationist

\section{Introduction}

An interview-based study provided an enlightening approach in understanding the information needs of the attendings and fellows at Memorial Sloan Kettering (MSK) Cancer Center's Breast Imaging Service. This is a niche group within the larger Department of Radiology with unique needs and they often require that the information requested be delivered in easily digestible formats in their fastpaced work environment in support of patient care and other research endeavors. Designed in partnership between the groups' Informationist and senior leadership, this study's objectives included evaluating information needs, establishing a resource knowledge baseline of the participants,

\section{Corresponding author:}

Antonio P DeRosa, Memorial Sloan Kettering Cancer Center Library, 1275 York Ave, New York, NY I0065, USA.

Email: DeRosaAI@mskcc.org 
determining the existing ways through which the clinician's access evidence, and examining any gaps in the current services being offered. The goal was to diagnose what could be improved in order to enhance the information experience and deliver user-driven services.

The role of the Informationist, generally, consists of embedding an information professional into the clinical and/or research workflows of a specified group of individuals to enhance their information/knowledge management, evidence-based practice (EBP), and patient care practices. ${ }^{1}$ Although the title of Informationist may be relatively new, the idea behind placing a librarian with a clinical group goes back to the 1970s when Gertrude Lamb decided to move medical reference librarians out of the stacks and onto clinical services, creating what is known as clinical librarianship. ${ }^{2}$ Ms Lamb's legacy continues as the clinical librarian role exists in many academic and research library settings worldwide. ${ }^{3}$ The information services offered by these individuals over the years have led to the more modern - and domain knowledge-specific - role of the Informationist. The MSK Library offers a clinical medical librarian (CML) program which consists of the reference librarians acting in an Informationist capacity to a number of departments throughout the organization.

\section{Materials and methods}

A 10-point questionnaire (Appendix 1) was developed and used to interview 12 attending physicians and three fellows. Attending physicians are fellowship-trained breast imagers who are either on the research track $(n=3)$ or the clinical track $(n=9)$. Research track attendings are expected to perform independent research and publish articles. Both research and clinical track attendings are expected to perform clinical work, teach residents and fellows, and have administrative responsibilities. Breast imaging fellows have completed a residency in radiology and are obtaining a year of additional training that includes 9 months dedicated to clinical breast imaging. Breast fellows are responsible for the work in the clinic, several interdisciplinary conferences as well as a single independent research project during the training period. A two-part schematic (Appendix 2) was also created to capture more detailed feedback about the information needs and information-seeking behavior of clinicians regarding patient care (clinical) and research activities. This group of study participants was chosen at random and represents half of the Breast Imaging Service in the Department of Radiology at MSK.

Interviews took place in person and via phone or WebEx (virtual) for those participating from satellite locations. Interview questions were developed in collaboration with the Breast Imaging Service chief attending in an effort to keep the questionnaire relevant to this specialized group of clinicians. The study survey schematic was designed for the purpose of capturing more detailed feedback on the types of information sought and the participants' resources/knowledge-base (and behavior) used to locate the information. The schematic was either completed at the time of the interview (12 participants) for each individual or at a later time that was convenient for the clinician (3 participants).

\section{Results}

Clinical information needs vary among attendings and fellows in the Breast Imaging Service (Figure 1). Most attendings seek images of findings and procedural/technical imaging modality issues when it comes to their clinical practice. Fellows are seeking out challenging cases, most likely for weekly case conferences or journal clubs.

Taking a look at the ways in which attendings and fellows find the clinical information they seek (Figure 2), it is apparent that most attendings gravitate toward Google, PubMed, Radiologyspecific journals, and the library. Fellows tend to use Google and PubMed, but they also utilize a 


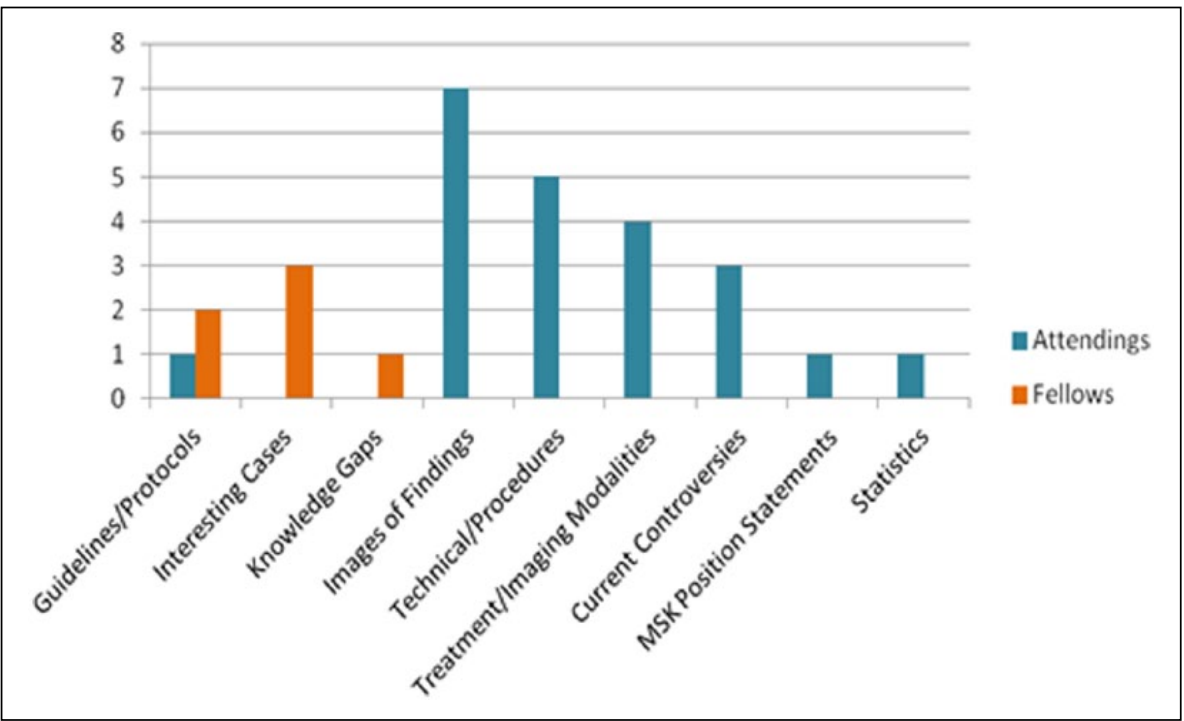

Figure I. Attendings versus fellows-clinical information needs (attending responses $=12$ and fellow responses $\mathbf{3}$ ).

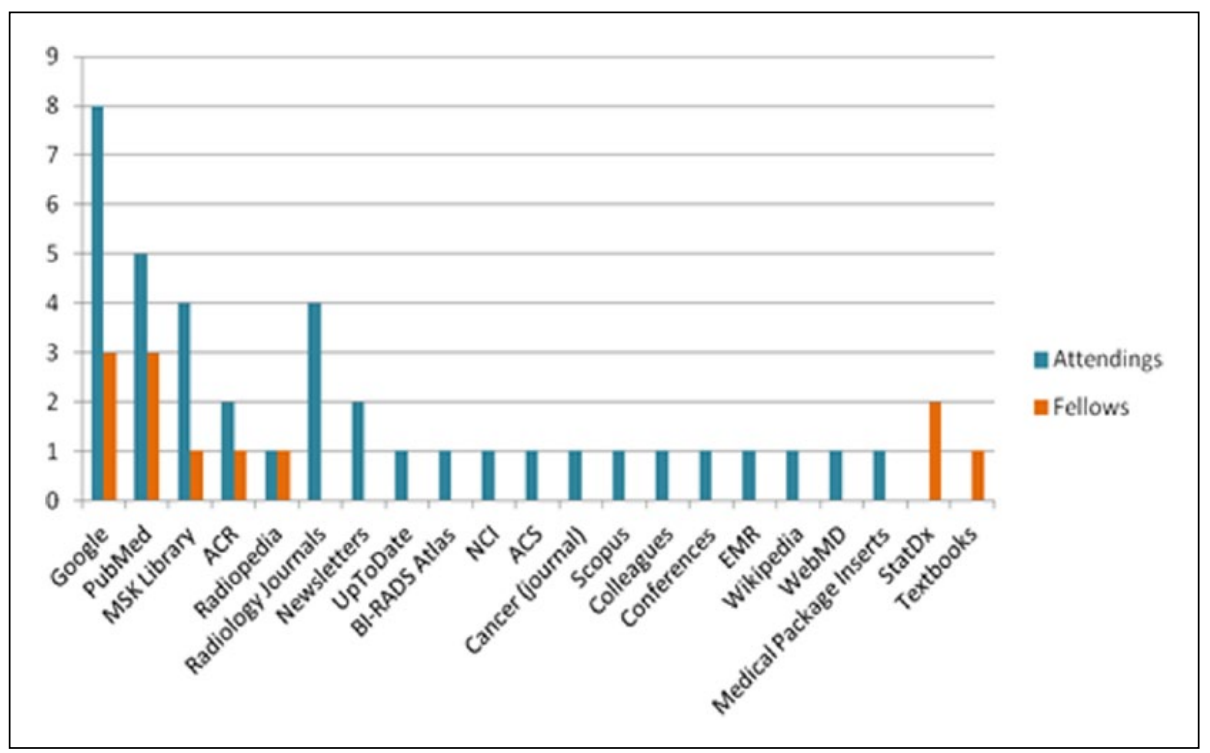

Figure 2. Attendings versus fellows - clinical resource knowledge base (attending responses $=12$ and fellow responses $=3$ ).

diagnostic imaging tool called StatDX while most attendings may not. None of the attendings interviewed mentioned this resource. StatDx is a diagnostic decision support system which provides access to peer-reviewed and non-peer-reviewed information on imaging cases in a variety of radiology sub-specialties. ${ }^{4}$ 


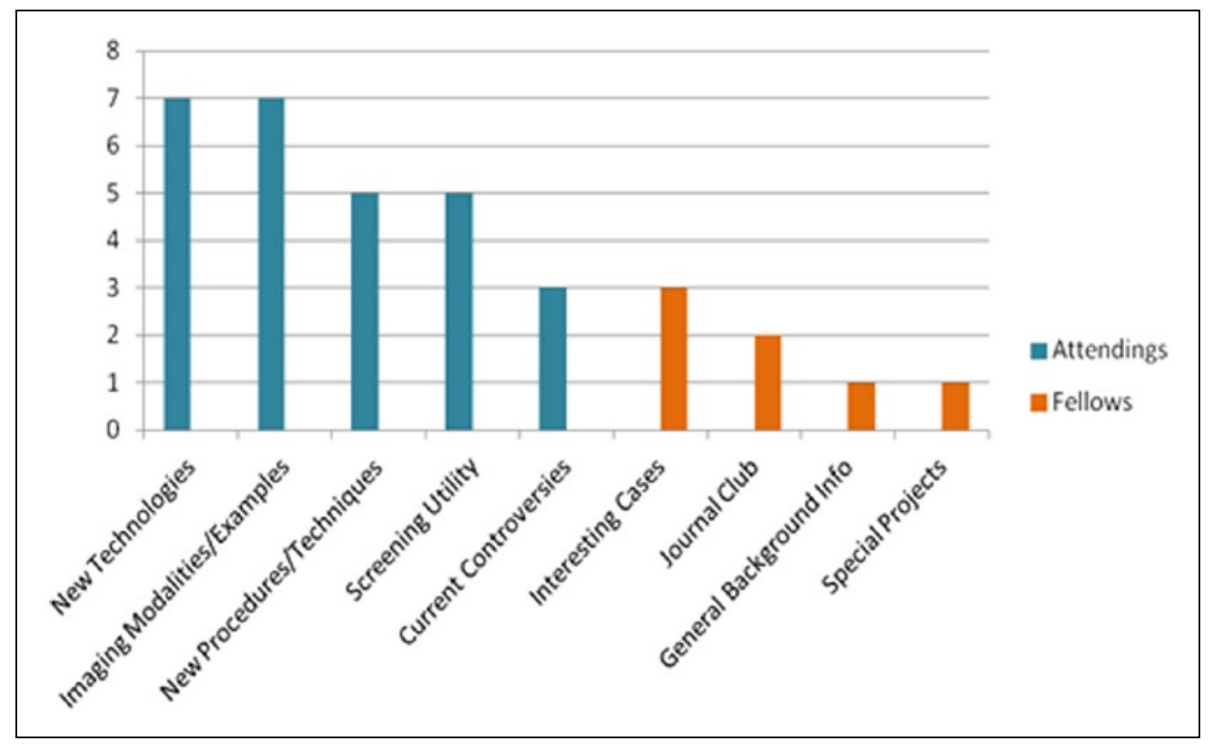

Figure 3. Attendings versus fellows-research information needs (attending responses $=12$ and fellow responses $=3$ ).

Regarding the research aspect of the Breast Imaging Service, the majority of attendings responded that they primarily conduct research to locate imaging modalities and new technologies in the field (Figure 3). Fellows, on the other hand, reported that their top research priority is to find interesting cases. Fellows responded similarly when asked what they search for during their clinical activities. Unlike the clinical information needs of both groups, the research needs offered no overlap in participant answers. That is, attendings and fellows as user groups had unique responses to the research information needs question.

When asked about their resource knowledge base involving a research project — or when generally conducting research - most attendings responded that the library or PubMed were their go-to sources for information discovery (Figure 4). Similarly, fellows also responded that the library and PubMed ranked at the top of their information resources list. Research in this context is used loosely. It includes all presentations (both internal and external), publications, conference articles, conference abstracts, and so on. Both groups conduct research for a variety of reasons, whether it is for personal awareness or for a professional endeavor.

\section{Bibliographic management tool use}

Another important aspect of both clinical practice and research practice is whether or not members of the Breast Imaging Service use a bibliographic management tool to curate and organize their publications/references with relative ease. EndNote was identified as the premier tool among the Breast Imaging Service with five attendings and two fellows using the program for citation management. Interestingly, only one individual (an attending) responded with Mendeley as a preferred tool of choice. Another noteworthy finding is that the National Center for Biotechnology Information (MyNCBI) was also mentioned by one attending, and a total of four individuals (one fellow and three attendings) responded that they don't use a bibliographic management tool at all. 


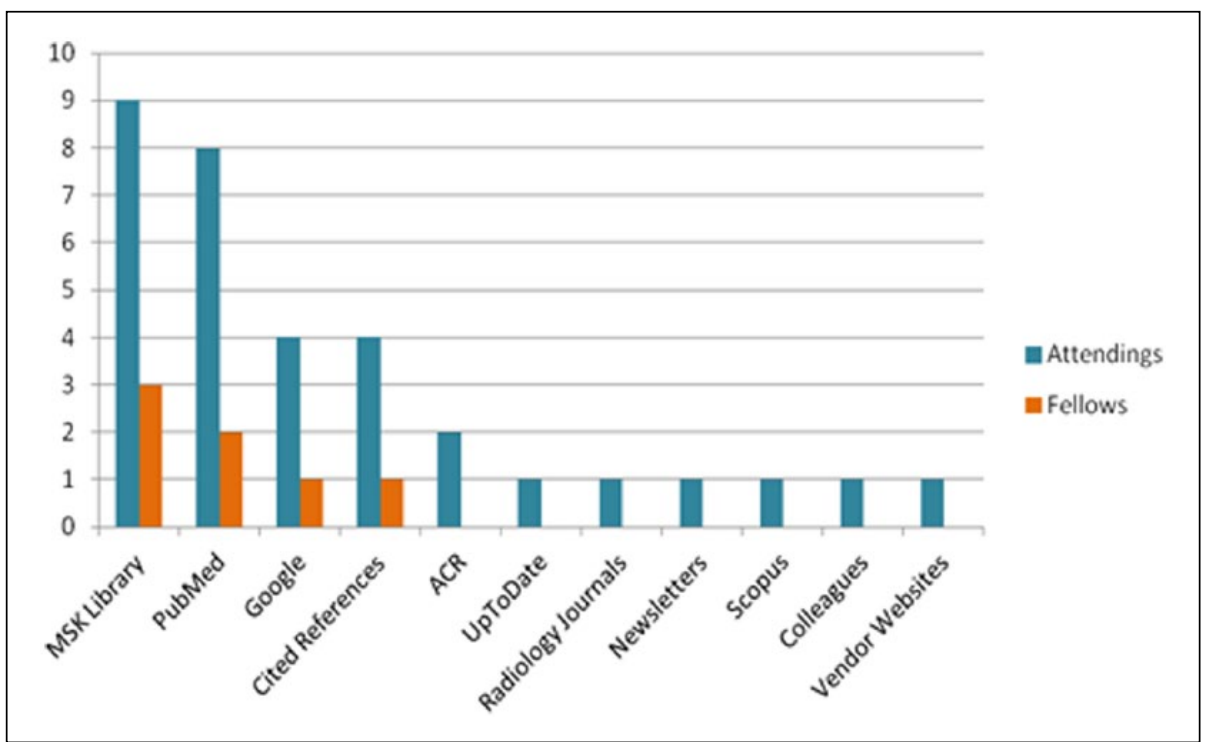

Figure 4. Attendings versus fellows - research resource knowledge base (attending responses $=12$ and fellow responses $=3$ ).

\section{Desired time versus actual time}

Time-related questions were incorporated into the interview process as a means of understanding just how long attendings and fellows spend searching for the information they need in both their clinical and research settings. Tables 1 and 2 show the desired time versus the actual time spent on finding clinical and research information, separated by role (attending and fellow).

The time ranges (averages) presented in these charts reveal a few things. Fellows tend to spend less time than desired searching for information on a typical patient case, while attendings actually spend more time than desired on a patient case. When it comes to researching a topic for a presentation, publication, conference, and so on, fellows seem to put in the amount of time they desired in a given week. Attendings, on the other hand, offered answers at opposite ends of the spectrum. On average, attendings would like up to $\sim 15 \mathrm{~h}$ a week for research purposes. The low range of attendings responded that the actual time put in on a weekly basis is up to $\sim 4 \mathrm{~h}$ and the high range of respondents cited spending up to $\sim 72 \mathrm{~h}$ a week conducting research. The two disparate ranges in actual research time put in by attendings is due to the fact that some of those clinicians interviewed have little focus on research projects - as they do not pertain to their job function. These are mostly attendings who work in a more clinically focused environment at a satellite location of MSK — not all radiologists are required to publish or present at national meetings.

Table I. Clinical questions-desired time versus actual time.

\begin{tabular}{|c|c|c|c|}
\hline \multicolumn{2}{|l|}{ Fellows } & \multicolumn{2}{|l|}{ Attendings } \\
\hline Desired & Actual & Desired & Actual \\
\hline$\sim 15 \mathrm{~min}$ & $\sim 2-3 \mathrm{~min}$ & $\sim 0-15 \mathrm{~min}$ & $\sim 0-30 \mathrm{~min}$ \\
\hline
\end{tabular}


Table 2. Research activities—desired time versus actual time.

\begin{tabular}{|c|c|c|c|}
\hline \multicolumn{2}{|l|}{ Fellows } & \multicolumn{2}{|c|}{ Attendings } \\
\hline Desired & Actual & Desired & Actual \\
\hline$\sim 1-4 h^{a}$ & $\sim 1-4 \mathrm{~h}^{\mathrm{b}}$ & $\sim 1-15 h^{\mathrm{a}}$ & $\begin{array}{l}\text { Low range: } \sim 1-4 \mathrm{~h}^{\mathrm{b}} \\
\text { High range: } \sim 15-72 \mathrm{~h}^{\mathrm{b}}\end{array}$ \\
\hline
\end{tabular}

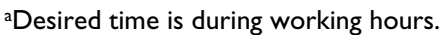

${ }^{\mathrm{b}}$ After-hours or weekend time.

Overall, both fellows and attendings would like to have more time during the work week devoted, specifically, to conducting research for publication or presentation.

\section{Discussion}

While the desire to spend more time on information-related activities is present, often the reality of work priorities and the focus on patient care limits the time that can be allocated in conducting topic searches. An Informationist can help to ease the research burden by working closely with his or her assigned team to deliver and organize the published information located in a way that permits the attending or fellow to continue to enhance their knowledge and expertise. ${ }^{5}$ Over time, the Informationist will develop a sixth sense in the topics that matter and can also proactively distribute published studies and other sources of information that will help support an ongoing learning environment and quality patient care.

Interpreting the responses from the questionnaire as well as the complete schematics from survey participants, an Informationist can contribute as a team member and collaborator by offering the much-needed support for clinical and research activities. One apparent way in which an Informationist may help a clinical group is by offering general consultation and training services, both on an individual and group level. Knowing the information needs of the attendings and the fellows will help to drive training opportunities.

Survey participants, regardless of status or experience, have their unique ways of locating information as well as their arsenal of go-to resources. Informationists can not only assist with the resource knowledge base that already exists within a group, but they can help to expand where these individuals seek information and offer an alternative perspective to research and EBP. Offering just-in-time resource training can enhance the research experience of the clinician as well as save them time and effort. New resources/databases can be introduced and various advanced and existing techniques for discovering information may prove useful to busy clinicians. ${ }^{6}$ Educating clinicians on targeted research methods and resources is a key role of the Informationist and one that has proven value. ${ }^{7}$ According to the survey results, both attendings and fellows tend to leverage the same resources in their research endeavors and so there is an opportunity for the Informationist to showcase other information resources that would support the workflow and research activities of these groups as well as keep them abreast of new features and enhancements in the tools that they use daily.

For example, based on the questionnaire results, 4 out of 15 individuals $(26.6 \%)$ responded that they do not use a bibliographic management tool to organize and curate their references when working on a research project. An Informationist can offer customized training sessions on specific tools or the importance of organizing references in general. Steps can also be taken to increase awareness about bibliographic management tools during individual consultation or other potential teaching moments by way of various on-campus meetings and conferences. 
When it comes to general research practices, current awareness alerts and notifications can be tailored for the individual and set-up by an Informationist for the ongoing learning of the clinician. This is especially true when it comes to the fellows as there is more of a research focus than clinical among this group - they are not usually as clinically oriented as the attendings in the service as they act in more of a learner capacity throughout their fellowships. Presenting search results from a number of different resources may bring varying perspectives to light as well as help to keep clinicians informed on topics important to them. ${ }^{8}$ Current awareness alerts can serve a number of different purposes. For instance, an alert created for the Breast Imaging Service helps to keep members in the know about current imaging controversies and new guidelines/policies from both the published literature (MEDLINE) and lay press (major newspapers, Auntminnie.com, New England Journal of Medicine (NEJM), Journal Watch, Medscape Radiology, etc.). The benefit of this level of service can span both the clinical and research spectrums. Clinicians may find pertinent information for use in a presentation, or they might simply utilize the current awareness alert as a discovery tool for understanding the current policies, procedures, and protocols in their field.

Informationists may also have the opportunity to insert themselves into the various clinical and research workflows of the group in which they are embedded. Time is always a factor when it comes to busy clinicians and so trusted information services can assist with guidance on overall productivity practices related to scoping the literature for evidence, as well as productivity practices related to technical workflows. ${ }^{9}$ For instance, iPad (and other tablet) personalization can be made to enhance the experience and productivity of both fellows and attendings. Introducing document annotation, bibliographic management, and note-taking apps on personal and professional devices may help to save time and effort when researching a topic, composing an article, or developing a presentation. The Informationist can be more than simply the gatekeeper and disseminator of information. ${ }^{10} \mathrm{~A}$ true partnership can take place between information professionals and clinical practitioners as a move away from the traditional transaction-based relationship between the librarian and clinician takes place. It is becoming increasingly important for clinicians to receive targeted and succinct information on-the-go and at the point of care and the Informationist is in an excellent position to support these needs. ${ }^{11}$

EBP is not only a buzz word among the medical community when it comes to patient care; it is now an imperative way of diagnosing and treating patients given the exponential volume of articles published each week. Most attendings and fellows feel confident in their ability to address difficult or unique patient care issues; staying updated with the literature is a critical way in which they maintain their level of expertise. It might be an added incentive for clinicians to participate in EBP training if it was offered in a self-paced capacity (e.g. video tutorials, short training documents, and LibGuides/research guides). ${ }^{12}$ The Informationist can support the clinician in point of care decision-making by retrieving the highest level of evidence possible. EBP training sessions can be implemented to offer clinicians the tools needed to perform complex searches and feel more confident with their results, ultimately to support their decisionmaking process. ${ }^{13}$ One-to-one consultation services and educational sessions on various EBP resources can help the clinician to save time, be more efficient, and offer high-quality decisionmaking regarding patient care.

Given where each group is in their career ladder, the differing responses to the questionnaire and schematic make sense. Throughout the fellowship, fellows tend to focus their efforts on learning the imaging techniques and established practice. ${ }^{14}$ Therefore, their research activities mainly consist of reading case studies on varying modalities or interesting cases on difficult-to-diagnose images. The attendings in the service have more experience and are more knowledgeable on the standard practices of care as well as interpreting images. Overall, the professional level, position, and role of the clinician influence their research- and information-based needs. 


\section{Conclusion}

The limited sample size of this study limits the generalizability of the survey and schematic results. However, the following take-home points can be drawn from the findings of this study:

- The role of the Informationist is an emerging, yet valuable one to assigned clinical groups;

- Clinician's knowledge-base, domain current awareness, productivity enhancement, and evidence-based care can be improved using Informationist services;

- An Informationist embedded in a clinical group's workflow can help clinicians to save time and spend their time more efficiently when it comes to research endeavors and patient care.

\section{Acknowledgement}

The authors would like to thank Sylvie Larsen, MLIS, for her assistance with the initial organization and discovery of insights of the data gathered.

\section{Declaration of Conflicting Interests}

The author(s) declared no potential conflicts of interest with respect to the research, authorship, and/or publication of this article.

\section{Funding}

The author(s) received no financial support for the research, authorship, and/or publication of this article.

\section{References}

1. Davidoff F and Florance V. The informationist: a new health profession? Ann Intern Med 2000; 132(12): 996-998.

2. Lamb G. A decade of clinical librarianship. Clin Librarian Q 1982; 1: 2-4.

3. Sullo E, Harrod T, Butera G, et al. Rethinking library service to distance education students: analyzing the embedded librarian model. Med Ref Serv Q 2012; 31(1): 25-33.

4. Niederhauser BD, Liaw K, McDonald RJ, et al. Pick up a book or "google it?" A survey of radiologist and trainee-preferred references and resources. J Digit Imaging 2014; 27(1): 26-32.

5. Stellrecht $\mathrm{E}$ and Chiarella $\mathrm{D}$. Targeted evolution of embedded librarian services: providing mobile reference and instruction services using iPads. Med Ref Serv $Q 2015$; 34(4): 397-406.

6. McLeod TG, Ebbert JO and Lymp JF. Survey assessment of personal digital assistant use among trainees and attending physicians. J Am Med Inform Assoc 2003; 10(6): 605-607.

7. Wagner KC and Byrd GD. Evaluating the effectiveness of clinical medical librarian programs: a systematic review of the literature. J Med Libr Assoc 2004; 92(1): 14-33.

8. Greyson D, Surette S, Dennett L, et al. "You're just one of the group when you're embedded": report from a mixed-method investigation of the research-embedded health librarian experience. J Med Libr Assoc 2013; 101(4): 287-297.

9. D'Alessandro MP, Nguyen BC and D'Alessandro DM. Information needs and information-seeking behaviors of on-call radiology residents. Acad Radiol 1999; 6(1): 16-21.

10. Wood GC. Serving the information needs of physicians. N Engl J Med 1972; 286(11): 603-604.

11. Shumaker D. The embedded librarian: innovative strategies for taking knowledge where it's needed. Medford, NJ: Information Today, Inc., 2012.

12. Putnam J, Faltermeier D, Riggs CJ, et al. Conquering evidence-based practice using an embedded librarian and online search tool. J Nurs Educ 2011; 50(1): 60.

13. Friedman C, Gatti G, Elstein A, et al. Are clinicians correct when they believe they are correct? Implications for medical decision support. Stud Health Technol Inform 2001; 84(Pt 1): 454-458.

14. Tabas G, Rubin F, Hanusa BH, et al. Expectations of groups versus pairs of attendings and residents about phone communications and bedside evaluation of hospitalized patients. Teach Learn Med 2005; 17(3): 217-227. 


\section{Appendix I}

\section{Survey questionnaire}

Information needs and behavior study—questionnaire

Interviewee:

Date:

Interviewer:

1. When researching a topic, condition, procedure, and so on for publication/presentation, where do you begin your search?

2. Why do you begin with this particular route?

3. When researching a topic, condition, procedure, and so on for patient care/decision-making, where do you begin your search?

4. Why do you begin with this particular route?

5. How do you keep yourself updated on the literature in your field? Please list sources/means of receiving current information.

6. Do you use a bibliographic management tool to organize and store your references while working on a research project?

7. If so, which tool do you use and do you feel as though you're taking full advantage of its capabilities?

8. Using your go-to resource/database for clinical management, please demonstrate how you would find information to help inform your decision-making on a patient's condition on stereotactic core needle biopsy and non-palpable breast lesions.

9. Are you satisfied with the results you retrieved?

10. How much time would you like to spend on clinical/patient care inquiries?

11. How much time, in reality, do you spend on searching for clinical/patient care information?

12. Using your go-to resource/database for research purposes, please demonstrate how you would find information on tomosynthesis screening in dense breasts.

13. Are you satisfied with the results you retrieved?

14. How much time would you like to spend on research inquiries?

15. How much time, in reality, do you spend on searching for information in your research field or area of interest? 


\section{Appendix 2}

\section{Completed survey schematic examples (attending and fellow)}

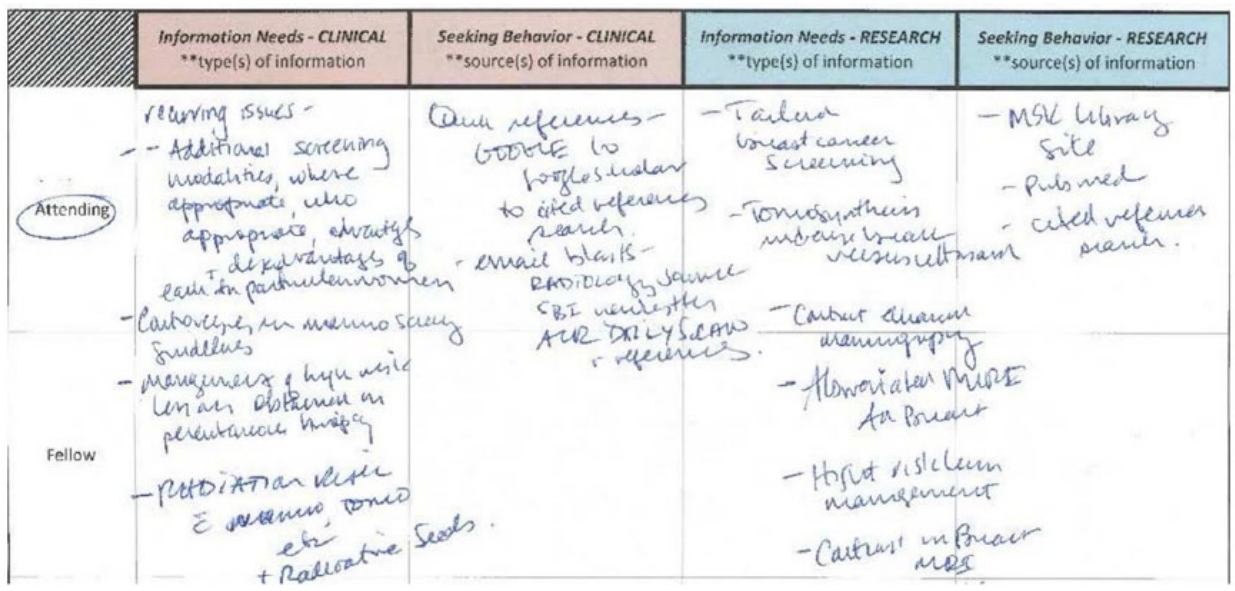

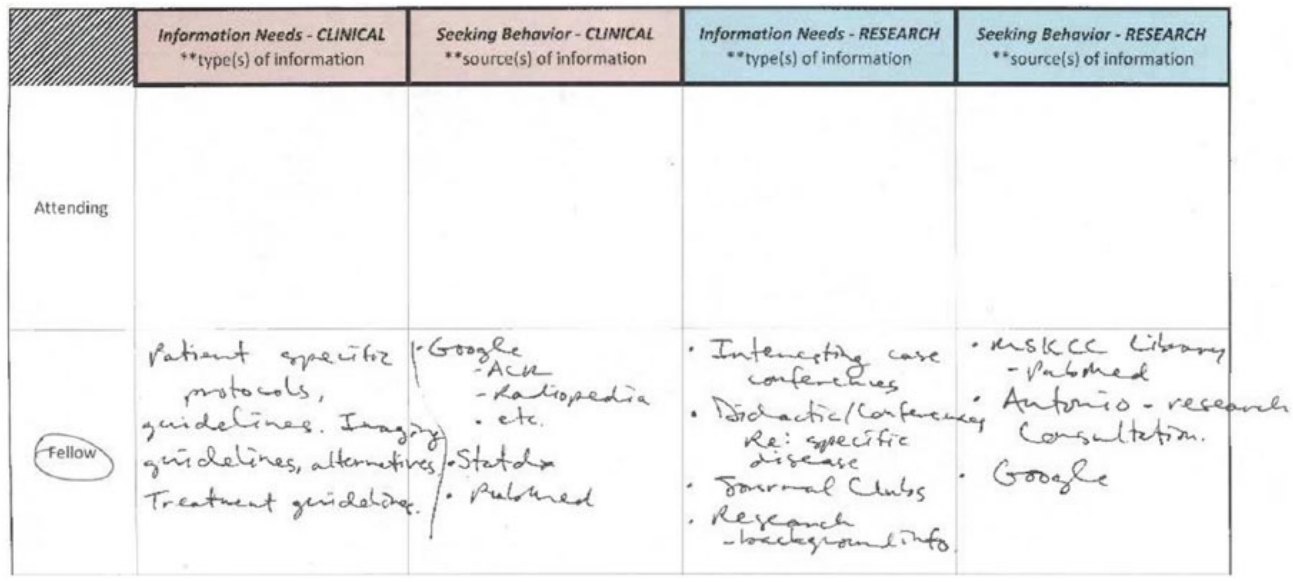

